\title{
Comparative Study of Widal and Typhidot Test in the Diagnosis of Typhoid Fever in Children
}

\section{Sreenivasa $\mathbf{B}^{1}$, Kumar $\mathbf{G V}^{2}$}

${ }^{1}$ Dr. B Sreenivasa, MBBS, MD, Associate Professor of Pediatrics, Basaveshwara Medical College, Chitradurga, Karnataka, India. ${ }^{2} \mathrm{Dr}$. GV Kumar, MBBS, DCH, DNB. Associate Professor of Pediatrics, Sri Siddhartha Medical College, Tumkur, Karnataka, India

\section{Address for correspondence:}

Dr. Kumar G.V (Associate Professor of Paediatrics),

Sri Siddhartha Medical College,

Agalakote, Tumkur, Karnataka, India. PIN572107

Tel No; +91

E-mail: kumargowripura@gmail.com

Acknowledgements: None

Funding: Nil

Conflict of Interest: None

Permission from IRB: Yes

Ethical dilemmas faced during study: No

\section{How to cite}

Sreenivasa B, Kumar GV. Comparative Study of Widal and Typhidot Test in the Diagnosis of Typhoid Fever in Children. J Nepal Paediatr Soc 2016;36(2):131-135.

doi: http://dx.doi.org/10.3126/jnps.v36i2.14761

This work is licensed under a Creative Commons Attribution 3.0 License.

\begin{abstract}
Introduction: Typhoid fever is a life threatening systemic infection that is usually presented with non- specific symptoms and signs. Its diagnosis is usually confirmed by blood culture and Widal test. The delayed results of microbiologic examination and the unreliable results of Widal test makes the search for a rapid and reliable method for diagnosis of typhoid fever. An early and accurate diagnosis is necessary for a prompt and effective treatment. The objectives were to compare the sensitivity and specificity of Widal test and typhidot with the blood culture in the diagnosis of typhoid fever in children.Material and Methods: A total of 200 hundred clinically suspected typhoid fever cases of 2-12 year age groups from both sexes attending Basaveshwara Medical College Hospital Chitradurga were studied. The blood collected aseptically was used for blood culture and clotted blood was used for Widal test and typhidot test. Results: Of 200 blood samples studied, 28 cases were positive by blood culture. Widal test showed sensitivity and specificity of $53 \%$ and $83 \%$ on blood culture proven cases. Typhidot test showed sensitivity and specificity of $93 \%$ and $83 \%$, respectively on blood culture proven cases. Conclusion: Using blood culture as the gold standard, typhidot showed a higher sensitivity compared to Widal test. The specificity of both Widal and typhidot remained the same. Typhidot could be a practical alternative to Widal test in the diagnosis of typhoid fever even in the resource poor laboratories.
\end{abstract}

Key words: Typhoid fever; Widal test; Typhidot test.

\section{Introduction}

$\mathrm{T}$ yphoid fever is a life threatening systemic infection occurring more frequently in developing countries where majority overcrowding and poor sanitation are prevalent and is a major public health problem ${ }^{1,2}$. It is estimated that more than 26.9 million typhoid fever cases occurs annually, of which $1 \%$ results in death. The vast of this disease burden is witnessed in $\mathrm{Asia}^{3,4}$ As the signs and symptoms of typhoid fever are non-specific, laboratory tests are essential for accurate diagnosis and early treatment with suitable antimicrobials for speedy recovery and prevention of complications and deaths and also for the control of transmission. The isolation of the organism from 
blood, bone marrow or stool is required to confirm the diagnosis ${ }^{5}$. Although the isolation of Salmonella Typhion blood culture remains the gold standard for diagnosing typhoid fever, it is time consuming, requires elaborate laboratory equipment and a level of technical expertise which may not be present in resource-poor laboratories. Even under best conditions, there may be failure to isolate the organism, especially after antimicrobial treatment has been started ${ }^{5}$.

Culture of bone marrow is more sensitive, but the procedure is invasive. Stool cultures are positive in only $30 \%$ of patients with the acute illness ${ }^{5}$. Widal test has been traditionally employed as a rapid serologic screening test but it remains a serological test with a moderate sensitivity and specificity. Molecular tests may achieve a high degree of accuracy, but the complexity of such assays and its high costs hinder its application other than as a research tool ${ }^{6}$ Therefore a rapid, inexpensive, reliable, easy to perform with high sensitivity and specificity serological test for diagnosis of typhoid fever which is appropriate for outpatient settings have been investigated to overcome the limitations of conventional methods

The present study was undertaken to compare the sensitivity and specificity of Widal test and typhidot with the blood culture in the diagnosis of typhoid fever.

\section{Materials and Methods}

The present study was conducted in the Department of Pediatrics, Basaveshwara Medical college Hospital Chitradurga from August 2014 to December 2015. Children between 2-12 years of age admitted with characteristic clinical features of typhoid fever such as fever ( $>38^{\circ} \mathrm{C}$ of $>4$ days duration), toxic appearance, headache, abdominal tenderness, hepato or splenomegaly, coated tongue, diarrhea or constipation were included in the study. Fever patients with alternative diagnosis were excluded from the study. Two hundred clinically suspected cases of typhoid fever of 2-12 years age groups of both sexes using simple random method constituted the study group. A minimum sample size of 200 cases were required using an incidence of typhoid fever as 156/2001 of total admissions at our institution at a significance level of 0.05 . Informed consent was obtained from all patients. Ethical committee clearance was obtained. All cases were subjected to a detailed history and thorough clinical examination. Following investigations were done on the day of admission. Complete blood count, typhidot test, blood culture and Widal test (for fever of $>7$ days duration).

The blood culture was done by using brain heart infusion (BHI) broth and it was incubated. Subsequent sub-cultures were made on Mac Conkey's agar and blood agar Medias after 24, 48 and 96 hours and the final sub-culture was made on the seventh day. The growth of S. typhi was identified by the standard biochemical tests and it was confirmed by agglutination with the salmonella polyvalent 'O', 09 and the $\mathrm{H}$ antisera. The Widal test was done by using the standard procedure (for $>7$ days fever) which was confirmed by the tube agglutination method and it was considered as positive when a titer of equal to or more than 1:160 was observed ${ }^{7}$. Typhidot testing was done on an acute serum (at first contact with the patient) by using the typhidot ${ }^{\circledR}$ (Malaysian BioDiagnostics Research SDN BHD) kit. It is a qualitative antibody detection test with total assay time of 1 hour ${ }^{8}$. The data was tabulated in Microsoft excel. Sensitivity, specificity, positive predictive value (PPV) and negative predictive value (NPV) were calculated.

\section{Results}

Out of 200 cases, $59.5 \%$ were male and $40.5 \%$ were females. The male to female ratio was $1.47: 1$. Out of 200 suspected cases, 28 (14\%) were positive by blood culture, $43(21.5 \%)$ by Widal(for patients with $>7$ days of fever) and $55(27.5 \%)$ by typhidot test (Table-1). Out of 28 blood culture positive cases which has taken as a gold standard was compared with the Widal test where only 15 showed positive results and 13 were negative (Table-2). The remaining 28 (of 43 cases) which were blood culture negative showed positive results with Widal test. Widal test has a sensitivity of $53 \%$, specificity of $83 \%$, and positive predictive value of $34 \%$ and negative predictive value of $91 \%$ in comparison with blood culture results (Table-4). Out of 28 blood culture positive cases which has taken as a gold standard was compared with the typhidot test where 26 showed positive results and only two cases were negative by typhidot (Table-3). The remaining 29 (of 55) which were blood culture negative showed positive results with typhidot test. Typhidot test has a sensitivity of $92 \%$, specificity of $83 \%$, and positive predictive value of $47 \%$ and negative predictive value of $98 \%$ in comparison with blood culture results (Table-4). 
Table 1: Results of Blood culture, Widal and Typhidot test.

\begin{tabular}{ccccccc}
\hline \multirow{2}{*}{ Results } & \multicolumn{2}{c}{ Blood culture } & \multicolumn{3}{c}{ Widal } & \multicolumn{3}{c}{ Typhidot } \\
\cline { 2 - 8 } & No. & $\%$ & No. & $\%$ & No. & $\%$ \\
\hline Positive & 28 & 14 & 43 & 21.5 & 55 & 27.5 \\
\hline Negative & 172 & 86 & 157 & 78.5 & 145 & 72.5 \\
\hline Total & $\mathbf{2 0 0}$ & $\mathbf{1 0 0}$ & $\mathbf{2 0 0}$ & $\mathbf{1 0 0}$ & $\mathbf{2 0 0}$ & $\mathbf{1 0 0}$ \\
\hline
\end{tabular}

Table 2: Comparison of Widal test with Blood culture

\begin{tabular}{|c|c|c|c|c|}
\hline & & \multicolumn{2}{|c|}{ Blood culture } & \multirow{2}{*}{ Total } \\
\hline & & Positive & Negative & \\
\hline \multirow{2}{*}{ Widal test } & Positive & 15 & 28 & 43 \\
\hline & Negative & 13 & 144 & 157 \\
\hline \multicolumn{2}{|c|}{ Total } & 28 & 172 & 200 \\
\hline
\end{tabular}

Table 3: Comparison of Typhidot with Blood culture

\begin{tabular}{|c|c|c|c|c|}
\hline & & \multicolumn{2}{|c|}{ Blood culture } & \multirow{2}{*}{ Total } \\
\hline & & Positive & Negative & \\
\hline \multirow{2}{*}{ Typhidot test } & Positive & 26 & 29 & 55 \\
\hline & Negative & 2 & 143 & 145 \\
\hline \multicolumn{2}{|c|}{ Total } & 28 & 172 & 200 \\
\hline
\end{tabular}

Table 4: Validity of Widal and Typhidot as a diagnostic tool in comparison with blood culture

\begin{tabular}{ccc}
\hline Parameter & Widal & Typhidot \\
\hline Sensitivity & 53 & 92 \\
\hline Specificity & 83 & 83 \\
\hline Predictive value of a positive test & 0.34 & 0.47 \\
\hline Predictive value of a negative test & 0.91 & 0.98 \\
\hline Likelihood ratio for positive test & 3.29 & 5.50 \\
\hline Likelihood ratio for negative test & 0.55 & 0.08 \\
\hline Accuracy & 0.795 & 0.845 \\
\hline
\end{tabular}

\section{Discussion}

Typhoid fever is a systemic illness with a significant morbidity and mortality in developing countries $^{9}$. Emergence of multidrug resistant strains of salmonella entericserovartyphihas only added to the burden of the disease. Any delay in diagnosis and inappropriate therapy increases the risk of outcome ${ }^{10}$. Blood culture has remained the gold standard for diagnosis of typhoid fever. In our study blood culture positivity among clinically suspected typhoid cases was in 28 cases (14\%). Culture positivity in other studies has quoted sensitivity ranging from $8.9 \%-43 \% 11,12,13$. The relative low rate of isolation from blood culture had been attributed to delay in diagnosis, widespread and irrational use of antibiotics and low volume of blood obtained for cultures among children. Widal test has been used for over a century in developing countries but its diagnostic utility has been limited due to low sensitivity, specificity and positive predictive value ${ }^{9}$. Decreased sensitivity is due to the long latent period after which the test may become positive. Decreased specificity is due to prior infection, vaccination with $T A B$ vaccine, cross reaction with other gram negative infections. In the present study, widely test was positive in $21.5 \%$ (43/200) of the patients. Widal test was positive in 15 of the 28 blood culture positive patients and 28 of blood culture negative patients. Thus the test had sensitivity of $53 \%$ and specificity of $83 \%$. Similar results have been reported in other studies from endemic areas ${ }^{9,11,14}$.

In a study by Mahaet $\mathrm{al}^{15}$ the sensitivity and specificity of Widal test were $81 \%$ and $71 \%$ respectively with 34\% PPV and 96\% NPV. Study done by Rahman et al. ${ }^{16}$ also reported, the sensitivity and specificity of the Widal test as $81 \%$ and $71 \%$ respectively. The interpretation of the Widal test remains problematic to this day, with a great number of articles reporting different cut-offs and the test has lost some popularity in recent years as technical skills are required for its 
performance and interpretation, different sensitivity and specificity rates are obtained even in same region ${ }^{17}$. Bakr et al. ${ }^{18}$ stated that different reports of sensitivity and specificity of Widal test are usually present due to different commercial antigens brands used.

In a developing country like India, the Widal test has been used extensively in the serodiagnosis of typhoid fever. However, Lateef A et al ${ }^{19}$ reviewed the significance of the Widal agglutination test and concluded that its use should not be encouraged in endemic areas. Ideally, in the Widal test, a fourfold rise of the antibody titer in paired sera is considered as diagnostic of typhoid fever. However, paired sera are often difficult to obtain and as a specific chemotherapy, it has to be instituted on the basis of a single Widal test only.

Typhidot test is based on detection of antibodies which appear in detectable titers as early as the second day of illness. It showed sensitivity of $92 \%$ and specificity of $83 \%$ in blood culture proved cases. This is in accordance with study done by Sherwalet $\mathrm{al}^{9}$ and Narayanappa et al ${ }^{11}$. Whereas in a study done by Zulfiqar et $\mathrm{al}^{20}$ sensitivity and specificity were $73 \%$ and $89 \%$ respectively. These differences may be due to several factors including the genomic diversity among S.typhi isolates in the region and differences in antigenic epitopes. Other factors responsible for reported differences in areas of high endemicity are various stages of the illness and the rate of increase of IgG antibodies to the outer membrane proteins, which may interfere with identification of concomitant IgM antibodies. In this study the typhidot test showed higher agreement with blood culture $(92.8 \%)$ than Widal test $(53.5 \%)$. This was also detected by Anushaet $\mathrm{al}^{21}$ as

\section{References}

1. Zulfiqar Ahmed Bhutta. Entric fever. In: Behrman RE, Kliegman RM, Jenson HB, editors. Nelson Textbook of Pediatrics. First south Asia edition. Philadlphia: Saunders Publishers; 2015. p. 1388-1393

2. Park's textbook of preventive and social medicine. 23 rd ed. Jabalpur: M/S BanarsidasBhanot; 2015. p. 234-238.

3. WorldHealth Organization. 6th International Conference on Typhoid Fever and other Salmonellosis. Geneva, WHO. 2006.

4. Crump JA, Stephen P, Luby ED. The global burden of typhoid fever. Bull World Health Org 2004;82:1-24.

5. Dutta S, Sur Dj, Manna B, Sen B, DebAK, Deen $\mathrm{JL}$, et al. Evaluation of new-generation serologic agreement of typhidot with blood culture has been calculated as (88\%).

The Typhidot offers an additional advantage among serologic diagnostic tests for typhoid fever in that the test strips do not require an ELISA reader for evaluation. The test was comparable to Widal test, but had a superior interpretative value, as it detects IgM antibodies thereby helps in early recognition of infection. The other advantage of this test was that it is less laborious, rapid and results are obtained within one hour. This will help the clinicians to initiate antimicrobial therapy at an early stage of the disease, thereby decreasing morbidity. Nevertheless, the higher cost of the test in comparison with the Widal test, as well as cold-storage requirements for test reagents, are additional impediments in using this test in developing country.

Large scale studies taking other serological tests as ELISA test for S.typhilgM or molecular diagnostic methods for diagnosis of detection of S.typhi infections as golden standards are highly recommended to overcome the drawbacks of blood culture low isolation rates that affects the sensitivity and specificity of the studied typhidot test. This helps to evaluate typhidot test more accurately compared to the traditional Widal test.

\section{Conclusion}

Even though similar such studies have been done in the past we reiterate that typhidot is still a practical alternative to other test in places where traditional culture facilities are not available for the diagnosis of typhoid fever on account of its increased sensitivity, early detection of cases, and ease of procedure with minimal infrastructure and availability of results on the same day.

tests for the diagnosis of typhoid fever: data from a community-based surveillance in Calcutta, India. DiagMicrobiol Infect Dis 2006;56(4):359-65.

6. Pastoor R, Hatta M, Abdoel TH, Smits HL. Simple, rapid and affordable point-of-care test for the serodiagnosis of typhoid fever. DiagnMicrobiol Infect Dis 2008;61:129-34.

7. Koneman, EW, Allen, SD, Janda WM, Schreckenberger PC, Winn WC. Colour atlas and textbook of diagnostic microbiology, $5^{\text {th }}$ edn. 1997. Lippincott, New York

8. Old DC. Salmonella infection. In: Collee, J.G., Fraster, A.G., Marmion, B.P., Simons, A. (Eds). Mackie and McCartney practical medical microbiology, 14th edn. Churchil Livingstone, New York.1996; 385402. 
9. Sherwal BL, Dhamija RK, Randhawa VS, Jais M, Kaintura A, Kumar M. A comparative study of Typhidot and Widal test in patients of Typhoid fever. JIndian AcadClinMed2004;5:244-46.

10. Begum.Z, Hossain, Md.A, Shamsuzzaman, A.K.M, Ahsan, Md.M., Musa, A.K.M. Mahmud, Md.C., et al.Evalutaion of typhidot (IgM) for early diagnosis of typhoid fever. Bangladesh J Med Microbio/2009; 3: 10- 13.

11. Narayanappa D, Sripathi R, Kumar JK, Rajani HS. Comparative study of Dot Enzyme Immunoassay (Typhidot-M) and Widal test in the diagnosis of Typhoid fever. Indian Pediatr 2009;47:331-33.

12. Jesudason MV, Sivakumar S. Propective evaluation of a rapid diagnostic test Typhidot for typhoid fever. Indian J Med Res 2006;123:513-16.

13. Saha MR, Duta P, Palit A, Dutt D, Bhattacharya MK, Mitra $U$, et al. A note on incidence of typhoid fever in diverse age groups in Kolkota, India. Jpn J Inf Dis 2003;56:121-22.

14. Khoharo HK. A comparative study of the Typhidot (Dot-EIA) and Widal test in blood culture positive cases of typhoid fever. Trop Doc2011;4:136-38.

15. Maha S. Hamd, Safaa Abdel-Rahman1, AdbelMgeed Mostafa, Sahar Abd-El Hameed. Evaluation of Enterocheck WB尺 test in Diagnosis of Typhoid
Fever among Egyptian Adults.Egyptian J Med Microbiol 2014;23(4):47-52.

16. Rahman M, Siddique AK, Tam FCH, Sharmin S, Rashid $\mathrm{H}$ lqbal A.Rapid detection of early typhoid fever in endemic community children by the TubexO9-antibody test. DaigMicrobiol Infect Dis 2007;58:275-81.

17. Wain J, Hosoglu S. The laboratory diagnosis of enteric fever. J Infect Developing Countries 2008; 2(6):421-425.

18. Bakr W, El Attar L, Ashour M, El Toukhy A.The dilemma of widal test- which brand to use? A study of fourdifferent widal brands: a cross sectional comparative study. Ann ClinMicrobio/Antimicrobio 2011; 8;10:7.

19. Lateef $A O$, King $A L$. The widal agglutination test- 100 years later: still plagued by controversy. Postgraduate Med J 2000;76:80-84.

20. Bhutta ZA, MansuraliN. Rapid Serologic Diagnosis of pediatric typhoid fever in an endemic area : A prospective comparative evaluation of two dotenzyme immunoassays and the widal test. Am J Trop Med Hyg 1999;61(4):654-57.

21. Anusha R, Ganesh R, Lalitha J. Comparison of a rapid commercial test, Enterocheck WB $(\circledR)$, with automated blood culture for diagnosis of typhoid fever.Ann Trop Paediatr 2011;31(3):231-34. 